\title{
Puntas de palmela: procesos tecnológicos y experimentación
}

\author{
Palmela points: technological processes and experimentation
}

\author{
Carmen Gutiérrez Sáez (*) \\ Alba López del Estal (*) \\ Álvaro Simón Martín (*) \\ Pedro Muñoz Moro (*) \\ Charles Bashore Acero (*)
}

\author{
Jorge Chamón Fernández $(*)$ \\ Ignacio Martín Lerma $(* *)$ \\ Elena Sanz Salas (*) \\ Ana Isabel Pardo Naranjo (*) \\ Juan Antonio Marín de Espinosa (***)
}

\section{RESUMEN}

La lectura funcional de las puntas de palmela ha generado cierta confusión. Se han interpretado como elementos de lanza, jabalina y flecha o exclusivamente como objetos de prestigio. Presentamos un trabajo experimental donde se evalúa la viabilidad de estas puntas en los tres tipos de armas. La colección incluye 36 puntas de palmela de cobre y bronce bajo en estaño, utilizadas sobre una presa, en pruebas de distancia y balística. Se trata también de determinar la función a partir de los contextos arqueológicos donde aparecen estas piezas. Los resultados arrojan luz sobre la posible utilización de estos objetos como armas eficaces.

\begin{abstract}
The function of palmela points has generated a certain confusion, being interpreted as the tips of a lance, javelin and of an arrow or exclusively as elements of prestige. The aim of this work is to present the results of experimentation on a prey with 36 palmela arrowheads of copper and low tin-bronze, in tests of distance and also of ballistics. It is a question of determining also the function from the archaeological contexts where these pieces appear. The results throw light on the possible utilization of these objects as effective weapon.
\end{abstract}

Palabras clave: Puntas de palmela; Arqueología experimental; Arqueometalurgia; Calcolítico; Edad del Bronce; Península Ibérica.

(*) Departamento de Prehistoria y Arqueología. Facultad de Filosofía y Letras. UAM. Cantoblanco. 28049 Madrid. Correo electrónico: carmen.gutierrez@uam.es

(**) Departamento de Prehistoria y Arqueología. Facultad de Humanidades. UNED. Senda del Rey 7. 28040 Madrid. Correo electrónico: imartin@bec.uned.es
Key words: Palmela point; Experimental Archaeology; Archaeometallurg; Chalcolithic; Bronze Age; Iberian Peninsula.

\section{INTRODUCCIÓN}

Las puntas de palmela son piezas representadas en los ajuares del Campaniforme de la Península Ibérica durante el Calcolítico tardío y el Bronce antiguo, que van siendo sustituidas por formas como las de pedúnculo y aletas durante el Bronce medio. Algunos ejemplares aparecidos en contextos más tardíos de la Edad del Bronce e incluso de la Edad del Hierro han sido interpretados como una perduración de objetos y no del tipo en sí mismo (Delibes y Fernández-Miranda 1981: 160; Rovira et al. 1992: 270; Rodríguez de la Esperanza 1999: 101; Herrán Martínez 2008: 233).

Forman parte de la panoplia de guerrero propia del mundo campaniforme, acompañando a puntas de flecha de formas variadas en sílex y puñales de lengüeta, puñales triangulares y alabardas de tipo Carrapatas en metal. Otros materiales asociados son adornos como gargantillas de oro, brazales de arquero, botones de perforación en $\mathrm{V}$ y cerámica campaniforme, generalmente de tipo Ciempozuelos (Delibes y Fernández-Miranda 1981: 158-159). Su distribución abarca toda la geografía peninsular, llegando, incluso, a suelo francés, si bien presentan concentraciones en la Submeseta Norte (147 ejemplares, Delibes et al.

$(* * *)$ Sílex Arqueología y Difusión del Patrimonio S.L. Calle Luz 30. Santo Ángel 30151. Murcia. Correo electrónico: info@tallarsilex.com

Recibido: 8-III-2010; aceptado: 12-IV-2010. 
1999: 68-69), Valencia (102 puntas de palmela, Simón 1998: 265) y el suroeste (76 puntas incluyendo triangulares y de pedúnculos y aletas, Hunt y Hurtado 1999: 299). En la Submeseta Norte, las puntas aparecen en yacimientos funerarios y de habitación, aquí de manera menos frecuente, aunque un elevado número de casos está sin contexto (Delibes et al. 1999: 78-79; Garrido Pena 2000: 173). Según avanza la Edad del Bronce su presencia en sitios funerarios va descendiendo progresivamente, hecho que se ha relacionado con los cambios de ritual entre el Calcolítico y la Edad del Bronce "en los que el papel simbólico del arco y las flechas, asociado con el mundo de la caza, va perdiendo progresivamente importancia" (Káiser 2003: 86).

Las interpretaciones acerca de la función de estos objetos son distintas. Delibes (1977: 109) propone el uso como punta de jabalina, o incluso de lanza, de los ejemplares de Fuente Olmedo "pues perderían en pocos metros la trayectoria de lanzamiento debido a su considerable peso". Son de la misma opinión, Briard y Mohen (1983: 117-118), Méndez Madariaga (1997: 65), Fernández Manzano y Montero (1997: 112) y Garrido Pena (2000: 211). De forma similar, Káiser (2003: 79 y 87) las interpreta como puntas arrojadizas, de dardo o azagaya, insistiendo en su elevado tamaño y peso. Rovira Port (1998: 127-130) las define directamente como puntas de jabalina.

Simón (1998, 1999: 204) las clasifica como puntas de flecha, si bien destaca su aparición conjunta con ejemplares en sílex durante el Campaniforme. Káiser (2003) no lo descarta para las puntas más pequeñas. Sin aludir a su tamaño, Hernando Grande (1992) las incluye también en esta categoría. La posición más firme a favor de esta interpretación es mantenida por Blas Cortina y Rovira Llorens (2005: 290-292).

Finalmente, algunos autores se inclinan por asignarles un valor más simbólico que funcional debido a las deficientes características mecánicas del cobre frente al bronce (Delibes y Santiago 1997: 103, 107; Fernández Manzano y Montero 1997: 111-113).

\section{ASPECTOS MORFOLÓGICOS, FUNCIONALES Y CRONOLÓGICOS}

La morfología básica de estas piezas es bastante homogénea. Consiste en una punta más o menos ovalada que se prolonga en un pedúnculo de longitud diversa. Las variaciones de esta forma combinan dos aspectos, la morfología de la hoja (de ovalada a romboidal) y su prolongación en un pedúnculo a partir de la anchura máxima. Esta unión puede hacerse suavemente o de forma más marcada mediante muescas.

Combinando estos criterios, Delibes (1977: 110) ha definido tres tipos básicos: A de forma oval, $\mathrm{B}$ con pedúnculo estrangulado por dos muescas y $\mathrm{C}$ de forma romboidal y ancho pedúnculo. Los dos primeros presentan, a su vez, variaciones relacionadas con la longitud del pedúnculo. No es raro que distintos tipos aparezcan conjuntamente, como señala el propio Delibes (1977: 111), en el ajuar de Fuente Olmedo. A estas tres formas generales añaden Rovira et al. (1992) dos nuevos elementos, los tipos D, de morfología triangular, y E, la clásica punta de aletas con pedicelo prolongado, que aparece ya en el Bronce antiguo pero arraiga principalmente en el Bronce pleno.

La materia prima empleada en estas piezas es el cobre o el cobre arsenicado. Las diferentes morfologías de las puntas (A, B y C) parecen correlacionarse con el desarrollo de tratamientos como el recocido, poco habitual en la forma A y más frecuente en los otros dos tipos. Este hecho, según Rovira et al. (1992: 278), podría indicar que el tipo A, el más común, es también el más antiguo. Del mismo modo, la consolidación de puntas como las de pedúnculo y aletas durante el Bronce medio se relacionaría con avances metalúrgicos como las aleaciones de estaño. Por su parte, los resultados del análisis de multivariantes sobre 108 puntas de palmela de la Meseta, inducen a Garrido Pena (2000: 179) a considerarlo un tipo metálico fuertemente estandarizado cuya evolución gradual está relacionada con el sistema de enmangue.

A este respecto, Simón detalla un progreso en la forma de las palmelas. En las más antiguas, durante el Campaniforme, entre finales del III milenio e inicios del II, se prima la anchura. Serían de hoja ovalada con unión semicircular y pedúnculo cuadrangular tendente a largo. Las formas clásicas, a partir del Bronce antiguo, tendrían la zona de unión ovalada en una pieza estilizada donde se favorece la longitud. Estas últimas convivirían a lo largo del II milenio con palmelas o foliáceas de hoja triangular y unión trapezoidal (Simón 1998: 265). La sección de la hoja es comúnmente lenticular con un espesor apenas más desarrollado en 
la parte central. No es frecuente la presencia de nervio central ni siquiera de un engrosamiento apreciable en la hoja, que proporcionarían robustez evitando que se doblase en un impacto, aunque es patente en algunos ejemplares de las colecciones de Celada de Roblecedo, Carrión de los Condes y Palencia (Delibes y Fernández-Miranda 1981: 165-166, Figs. 2 y 5), Acebuchal (Hunt y Hurtado 1999: Fig. 8), Alcañiz (Gabaldón et al. 2006: Fig. 1) o el inédito de Coca (J.F. Blanco comunicación personal), (Fig. 1), entre pocos más. Este atributo aparece en la Edad del Bronce antiguo y se generaliza, en otros tipos de puntas, en el Bronce final (Káiser: 2003: 79 y 88).

A pesar de la escasa diversidad morfológica que facilita el reconocimiento de estas puntas, en el registro arqueológico se observa un fuerte abanico de tamaños y pesos, según se desprende de la

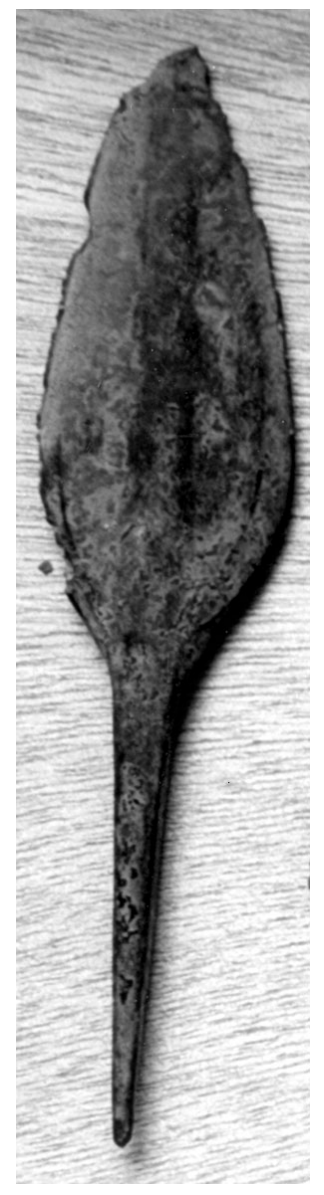

Fig. 1. Punta palmela con grueso nervio central. Coca, inédita. relación de materiales del Proyecto de Arqueometalurgia de la Península Ibérica (Rovira et al. 1997). La longitud de las puntas de palmela oscila entre 38 y $170 \mathrm{~mm}$, alcanzando $180 \mathrm{~mm}$ en el ejemplar de Bullas (Murcia). Las anchuras están comprendidas entre los $11 \mathrm{~mm}$ de las de Santa María del Retamar (Ciudad Real) y El Acebuchal (Sevilla) hasta los $37 \mathrm{~mm}$ de algunas puntas de Fuente Olmedo (Valladolid), siendo una anomalía los 2 ó $3 \mathrm{~mm}$ de los ejemplares del Cocherón y Prado de la Nava (en Sevilla y Salamanca respectivamente). El peso de estos ejemplares se reduce a unos 4 gr para las piezas más ligeras y alcanza entre 35 y 38 gr en las de la colección Blanco Cicerón de A Coruña. Por debajo de los pesos mínimos está la pieza del Monumento 2 de Parxubeira en A Coruña.

Esta misma variedad de tamaños ha sido constatada por Garrido Pena en su estudio de 107 ejemplares de la Meseta. El grueso de las puntas $(78,10 \%)$ se hallan comprendidas entre 7 a $12,5 \mathrm{~cm}$ de longitud y 1,4 a $3,7 \mathrm{~cm}$ de anchura, si bien constata una concentración significativa $(39,41 \%)$ de elementos entre $8-10 \mathrm{~cm}$ de longitud y 1,7-3,3 cm de anchura. El grosor y el peso se han estudiado a partir de un menor número de piezas (20 y 33 respectivamente) ofreciendo el primero una media de $2,6 \mathrm{~mm}$ de un rango que oscila entre 2 y $4 \mathrm{~mm}$. El peso, por su parte, se distribuye entre 5,63 y $31,8 \mathrm{gr}$, con un promedio de 17,6 gr, si bien en el histograma adjunto, se observa que el mayor número de ejemplares corresponde a puntas pesadas entre 20,1 y 25 gr (Garrido Pena 2000: 174-175).

Como hemos mencionado previamente, la morfología general de las puntas de palmela es relativamente uniforme, haciendo posible su rápido reconocimiento, pero su variación en peso y tamaño no facilita determinar su función.

\section{LOS EXPERIMENTOS}

Este trabajo experimental se plantea con un doble objetivo. El primero es contrastar la eficacia del cobre y bronce bajo en estaño como materias primas de puntas arrojadizas. Para ello se han llevado a cabo cadenas tecnológicas de forja, forja en frío y recocido en la elaboración de las puntas. Éstas han sido empleadas sobre una presa, valorándose su capacidad de penetración y deformación. El segundo es probar su viabilidad como armas en los distintos tipos propuestos: flechas, 
lanzas y jabalinas. En este caso, además de los experimentos anteriores, se llevaron a cabo pruebas de distancia y de balística con un medidor de velocidad registrando los metros por segundo del disparo con arco.

La muestra comprende 36 puntas de palmela, 24 de ellas se usaron en pruebas de penetración y deformación contra una oveja muerta, 4 en el estudio balístico y 8 en experimentos de distancia. Fueron manufacturadas y usadas en programas sucesivos, lo que nos permitió ir mejorando su eficacia a tenor de los resultados conseguidos.

\section{Tecnología. El proceso metalúrgico}

Los estudios de composición de las puntas de palmela arqueológicas nos muestran una presencia mayoritaria de cobres con pequeñas impurezas, aunque no faltan piezas con proporciones de arsénico que no suelen superar el $2 \%$ (Rovira et al. 1997). Por este motivo, nuestra colección se ha elaborado sobre cobres puros y bronces con un $2 \%$ de estaño. La elección del estaño se debe a la dificultad de experimentar con arsénico y al hecho de que las propiedades mecánicas de los cobres arsenicales y los bronces con aleaciones bajas en estaño son equiparables (Northover 1998).

Sólo una de las 24 puntas de palmela del primer experimento fue forjada en frío a partir de una lámina de bronce con $2 \%$ de estaño fundida por nosotros mismos. El resto fueron batidas sobre chapa de cobre puro hasta conseguir un espesor de $3 \mathrm{~mm}$. De este conjunto, 8 puntas fueron recocidas en horno durante 1 hora a $600^{\circ}$, tras previa forja. Una vez terminados estos procesos se limaron y afilaron para conseguir el acabado final.

Para el siguiente grupo de experimentos (12 puntas) se partió tanto de las chapas de cobre puro como de dos láminas de bronce (al $2 \%$ de estaño) con $6 \mathrm{~mm}$ de grosor medio, obtenidas fundiendo $1 \mathrm{~kg}$ de cobre puro en granalla y $20 \mathrm{gr}$ de estaño en polvo y moldeadas en moldes de arena (Gutiérrez y Soriano 2008). Inicialmente, tratamos de producir puntas de palmela, media- nas y grandes, directamente por moldeado. Los resultados fueron problemáticos ya que el metal era poco fluido, se distribuía mal por los moldes y se requería más cantidad en el vertido. Las piezas resultaron anormalmente gruesas superando pesos de 100 gr. Convertir esas piezas en puntas más ligeras, mediante martilleado en frío y recorte, fue prácticamente imposible dadas las profundas fisuras que surgieron en el material por efecto de la tensión acumulada (acritud). El batido de las láminas de bronce presentó una dificultad similar pues hubo que reducirlas $2 / 3$, lo que provocó algunas fisuras y la necesidad de remodelar ligeramente las morfologías. De esta segunda colección experimental se reservaron 4 piezas para el estudio de balística.

Los ejemplares fabricados son de hoja lanceolada con pedúnculo fino y largo. La sección de la hoja es lenticular y la del pedicelo rectangular. El conjunto ha sido clasificado en tres módulos determinados por su peso y tamaño (Tab. 1).

Las puntas se han utilizado como flecha, lanza y jabalina, evaluando su viabilidad en cada una de las modalidades

\section{Las armas}

El arco. Los ejemplares de cronología más próximos al Campaniforme proceden de Meare Heath y Ashcott Heath (Somerset, Inglaterra). Son dos arcos de madera de tejo de 190 y $160 \mathrm{~cm}$ de longitud, un arco plano y otro de sección en $\mathrm{D}$ respectivamente (Clark 1963). Más conocido es el arco simple de Otzi, igualmente de tejo, que mide $182 \mathrm{~cm}$ de longitud y alcanza unas 40 libras de potencia, que nos lleva al inicio de la metalurgia (Spindler 1995). En el mismo tipo de madera se han construido los arcos de Charavines, si bien durante el Mesolítico se han documentado ejemplares en madera de olmo -Homelgaard- y fresno -Brabands (Muñoz y Ripoll 2006).

En nuestro caso hemos partido de una madera joven de fresno europeo (Fraxinus excelsior $L$ ) talado en enero de 2008 que se mantuvo secando

\begin{tabular}{|l|c|c|c|c|c|}
\hline & N. $^{\circ}$ total & \multicolumn{1}{|c|}{ Longitud } & Anchura & Espesor & Peso \\
\hline Pequeñas & 13 & $40-50 \mathrm{~mm}$ & $12-15 \mathrm{~mm}$ & $0,8-1,8 \mathrm{~mm}$ & $3,5 \mathrm{a} 6 \mathrm{gr}$ \\
Medianas & 15 & $90-115 \mathrm{~mm}$ & $20-28 \mathrm{~mm}$ & $1,8-2,8 \mathrm{~mm}$ & $18 \mathrm{a} 22 \mathrm{gr}$ \\
Grandes & 9 & $130-150 \mathrm{~mm}$ & $30-40 \mathrm{~mm}$ & $1,5-2,5 \mathrm{~mm}$ & $30-41 \mathrm{gr}$ \\
\hline
\end{tabular}

Tab. 1. Módulos de peso y tamaña de las puntas de palmela

T. P., 67, N. ${ }^{\circ} 2$, julio-diciembre 2010, pp. 405-418, ISSN: 0082-5638

doi: $10.3989 /$ tp.2010.10047 
durante un período de 8 meses. El tronco se seccionó longitudinalmente en dos mitades mediante cuñas de asta de ciervo y madera, seleccionándose una de ellas por su morfología y menor nudosidad. La corteza de la cara dorsal fue retirada intentando no seccionar los nudos, mientras que la cara ventral se trabajó hasta conseguir una sección semicircular con tensión uniforme. Como cuerda se ha empleado una trenzada de lino. Se ha conseguido así un arco simple de $178 \mathrm{~cm}$ de longitud y una potencia de 35 libras.

Las flechas. Como astiles hemos empleado plumas y varillas de madera de abeto. Las flechas miden $81,5 \mathrm{~cm}$ de longitud por $1 \mathrm{~cm}$ de grosor. Han sido emplumadas con tres plumas de oca de $10 \mathrm{~cm}$ de longitud y se ha abierto en el extremo superior una caja profunda para embutir los pedicelos. Para fijar las puntas se ha empleado tripa de cordero en las pequeñas y cuerda de lino en las medianas, resultando este último sistema mucho más seguro, ya que las tripas se soltaban con más facilidad. Hemos descartado el uso de almácigas como resina de abedul para evitar problemas en la limpieza y posterior observación de las huellas. No obstante, este sistema permite una fijación excelente. El

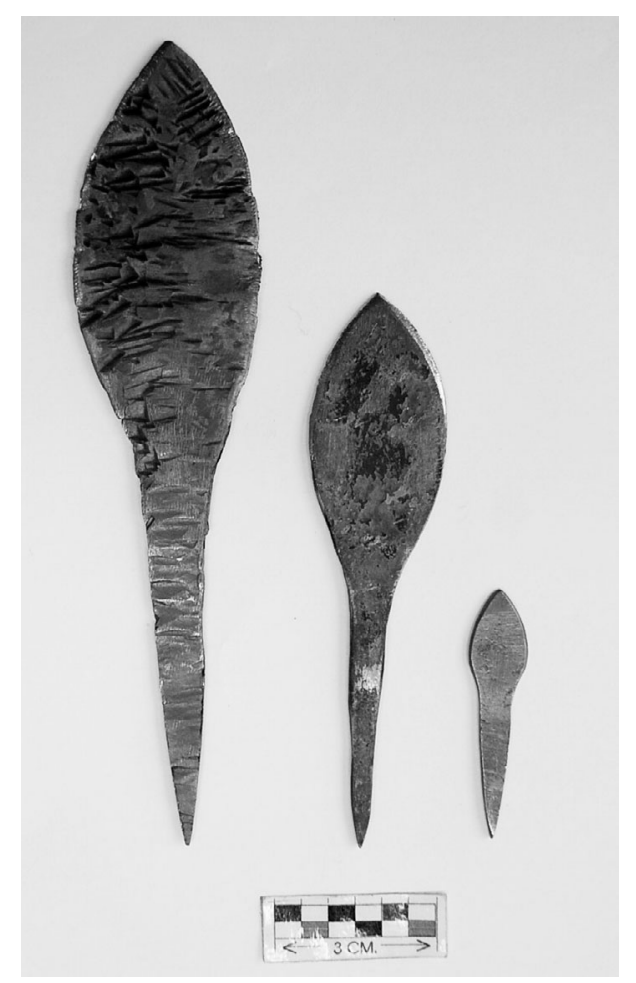

Fig. 2.A. Los tres tamaños de puntas de palmela. peso medio de las flechas armadas es de 56 gr para las puntas pequeñas y de 71 gr para las medianas.

Las jabalinas y las lanzas. Fueron montadas sobre vástagos de madera de pino de $163 \times 2 \mathrm{~cm}$ las primeras y $200 \times 2,8 \mathrm{~cm}$ las segundas. En ambos casos se practicó una abertura en caja en la zona superior para insertar los pedúnculos de las puntas. Éstas fueron fijadas, además, con cuerda de lino. Los pesos medios de jabalinas y lanzas montadas fueron de 346 gr para la jabalina con punta media y 658 para la lanza con punta grande (Fig. 2: A, B, C).

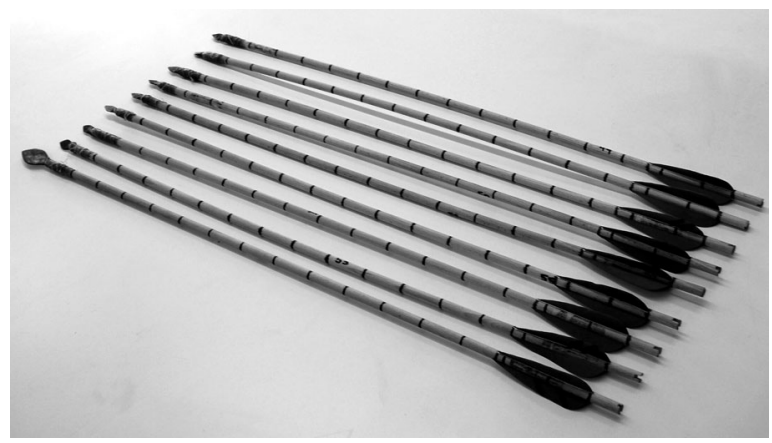

Fig. 2.B. Parte de las puntas de flecha montadas.

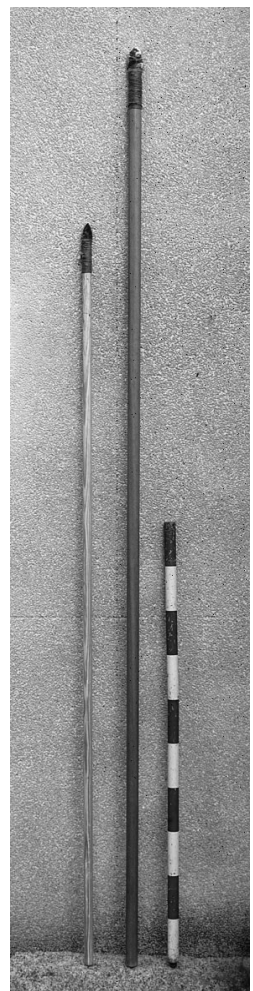

Fig. 2.C. Lanza y jabalina. 


\section{RESULTADOS}

\section{Pruebas de penetración y deformación}

Los resultados de tiro con puntas de flecha, jabalinas y lanzas sobre una oveja muerta quedan sintetizados en la Tabla 2.

Los resultados de las puntas son variables atendiendo a distintos factores. El primero es la técnica de fabricación, las piezas menos eficaces fueron aquellas que habían sido recocidas después de la forja en frío y no volvieron a ser batidas. Estas puntas, más blandas, se doblaron con facilidad, quedando inutilizadas después del pri- mer impacto en el animal o en madera (n. $\left.{ }^{\circ} 1\right)$, si bien las que obtuvieron disparos fallidos o sólo rebotaron pudieron ser reutilizadas unas pocas veces más (Fig. 3: A, B, C, D, E).

Otro factor significativo fue el sistema de enmangue. Aquellas puntas que fueron enmangadas hasta el punto de unión entre la hoja y la espiga se doblaron igualmente con facilidad en esta zona, sobre todo las recocidas. Para evitar este problema, las puntas siguientes fueron desmontadas y encajadas en el vástago de madera hasta el tercio basal de la hoja, revelándose así más duraderas. Las piezas fueron dejadas de usar al doblarse, caso de las recocidas, o al desprenderse del astil,

\begin{tabular}{|c|c|c|c|c|c|c|c|}
\hline N. ${ }^{\circ}$ & $\begin{array}{c}\text { Materia } \\
\text { Prima }\end{array}$ & $\begin{array}{c}\text { Tratami } \\
\text { ento }\end{array}$ & Tamaño & $\begin{array}{l}\text { Peso } \\
\text { (gr) }\end{array}$ & Arma & $\begin{array}{c}\text { Total } \\
\text { disparos }\end{array}$ & Resultado de los disparos \\
\hline 1 & $\mathrm{Cu}$ & $\mathrm{FF}+\mathrm{R}$ & Pequeña & 4 & Flecha & 1 & 1 clavada (madera) \\
\hline 2 & $\mathrm{Cu}$ & $\mathrm{FF}+\mathrm{R}$ & Pequeña & 4 & Flecha & 2 & $\begin{array}{l}1 \text { clavada (animal) } \\
1 \text { rebote }\end{array}$ \\
\hline 3 & $\mathrm{Cu}$ & $\mathrm{FF}+\mathrm{R}$ & Pequeña & 4 & Flecha & 1 & 1 clavada (animal) \\
\hline 4 & $\mathrm{Cu}$ & $\mathrm{FF}+\mathrm{R}$ & Pequeña & 3,8 & Flecha & 1 & 1 fallida \\
\hline 5 & $\mathrm{Cu}$ & $\mathrm{FF}$ & Pequeña & 4 & Flecha & 1 & 1 fallida \\
\hline 6 & $\mathrm{Cu}$ & FF & Pequeña & 4,2 & Flecha & 3 & 3 clavadas (animal) \\
\hline 7 & $\mathrm{Cu}$ & $\mathrm{FF}$ & Pequeña & 4 & Flecha & $10 *$ & $\begin{array}{c}6 \text { clavadas (animal), } 2 \text { rebotes } \\
2 \text { fallidas }\end{array}$ \\
\hline 8 & $\mathrm{Cu}$ & $\mathrm{FF}$ & Pequeña & 4,6 & Flecha & 3 & 3 rebotes \\
\hline 9 & $\mathrm{Cu}$ & $\mathrm{FF}+\mathrm{R}$ & Mediana & 19 & Flecha & 3 & 2 fallidas, 1 rebote \\
\hline 10 & $\mathrm{Cu}$ & FF & Mediana & 20,6 & Flecha & $4^{*}$ & $\begin{array}{c}3 \text { clavadas (2 animal, } 1 \text { madera) } \\
1 \text { fallida }\end{array}$ \\
\hline 11 & $\mathrm{Cu}$ & $\mathrm{FF}$ & Mediana & 21,9 & Flecha & 30 & $\begin{array}{c}5 \text { clavadas (animal), } 12 \text { rebotes } \\
13 \text { fallidas }\end{array}$ \\
\hline 12 & $\mathrm{Cu}$ & $\mathrm{FF}+\mathrm{R}$ & Mediana & 18,7 & Jabalina & 8 & 2 rebotes, 6 fallidas \\
\hline 13 & $\mathrm{Cu}$ & $\mathrm{FF}$ & Mediana & 18,5 & Jabalina & 6 & $\begin{array}{c}2 \text { clavadas (animal), } 3 \text { rebotes } \\
1 \text { fallida }\end{array}$ \\
\hline 14 & $\mathrm{Cu}$ & $\mathrm{FF}$ & Mediana & 20,1 & Jabalina & 10 & 7 rebotes, 3 fallidas \\
\hline 15 & $\mathrm{Cu}$ & FF & Mediana & 19,6 & Jabalina & 14 & $\begin{array}{c}2 \text { clavadas (animal), } 9 \text { rebotes } \\
3 \text { fallidas }\end{array}$ \\
\hline 16 & $\mathrm{Cu}$ & $\mathrm{FF}$ & Mediana & 18,7 & Jabalina & 6 & $\begin{array}{c}1 \text { clavada (animal y madera) } \\
4 \text { rebotes, } 1 \text { fallida }\end{array}$ \\
\hline 17 & $\mathrm{Cu}$ & FF & Grande & 35,1 & Jabalina & 8 & $\begin{array}{l}1 \text { clavada (madera), } 6 \text { rebotes } \\
1 \text { fallida }\end{array}$ \\
\hline 18 & $\operatorname{Br} 2 \%$ & $\mathrm{~F}+\mathrm{FF}$ & Mediana & 21,8 & Jabalina & 6 & $\begin{array}{c}2 \text { clavadas (madera), } 1 \text { rebote } \\
3 \text { fallidas }\end{array}$ \\
\hline 19 & $\mathrm{Cu}$ & $\mathrm{FF}$ & Mediana & 21,75 & Lanza & $10^{*}$ & $\begin{array}{c}5 \text { clavadas (animal), } 2 \text { rebotes } \\
3 \text { fallidas }\end{array}$ \\
\hline 20 & $\mathrm{Cu}$ & $\mathrm{FF}+\mathrm{R}$ & Grande & 30,5 & Lanza & 1 & 1 clavada (animal) \\
\hline 21 & $\mathrm{Cu}$ & $\mathrm{FF}+\mathrm{R}$ & Grande & 32,7 & Lanza & 1 & 1 clavada (animal) \\
\hline 22 & $\mathrm{Cu}$ & $\mathrm{FF}$ & Grande & 33,8 & Lanza & 2 & 1 clavada (animal), 1 rebote \\
\hline 23 & $\mathrm{Cu}$ & $\mathrm{FF}$ & Grande & 32,2 & Lanza & 2 & 1 clavada (animal), 1 rebote \\
\hline 24 & $\mathrm{Cu}$ & FF & Grande & 32,5 & Lanza & $25^{*}$ & 23 clavadas (animal), 2 rebotes \\
\hline
\end{tabular}

Tab. 2. Resultados de los experimentos sobre presa (Cu: Cobre, Br: Bronce, F: Fundido, FF: Forja en frío, R: Recocido). * Inclusión de peto en los disparos. 


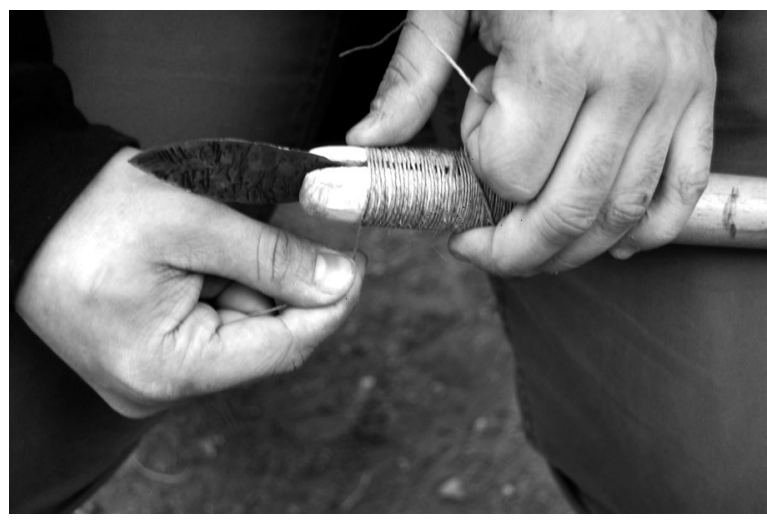

Fig. 3.A. Enmangado de una lanza con cuerda de lino.

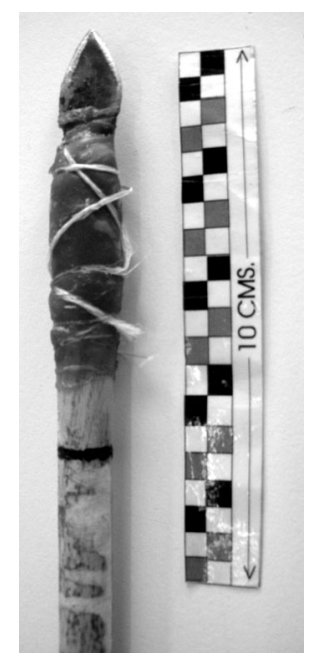

Fig. 3.B. Detalle de la punta de flecha 1, enmangada con tripas de cordero.
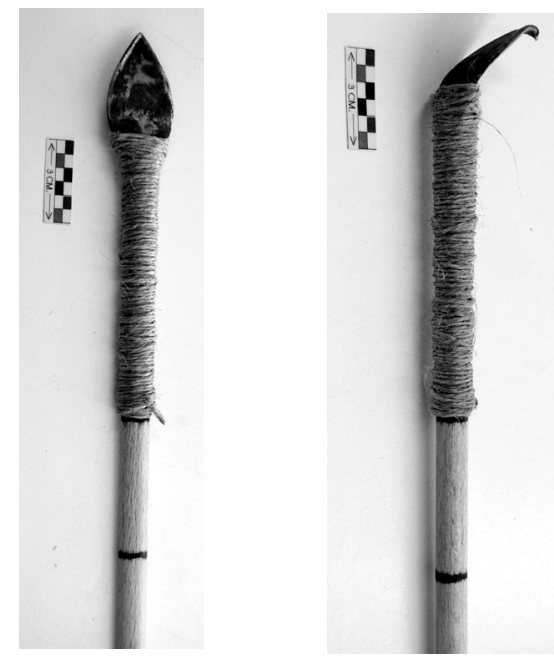

Fig. 3.C y 3.D. Punta de flecha 9 antes y después del uso.

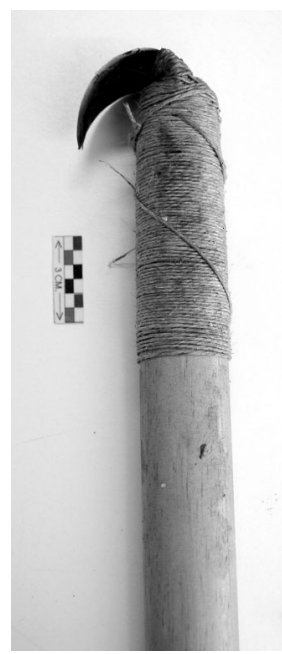

Fig. 3.E. Punta de lanza 20 después del uso.

circunstancia más común entre las que no sufrieron tratamiento térmico.

Las puntas que fueron trabajadas sólo mediante forja en frío, arrojaron resultados distintos. Se emplearon todas las flechas pequeñas y tres de las medianas, siendo óptimos los resultados de estas últimas en iguales condiciones de tiro. La distancia de tiro con arco fue de 3 a $5 \mathrm{~m}$ a excepción de la punta 9 (mediana) que fue disparada a $20 \mathrm{~m}$ de distancia. Con las flechas 7 y 10 y la lanza 19 se interpuso un pequeño peto de cuero de $2 \mathrm{~mm}$ sobre la oveja. En casi todos los casos las puntas consiguieron traspasar el cuero en varios de los disparos. Resultaron excepcionales las puntas 7 (pequeña) y 11 (mediana), por la cantidad de disparos que admitieron, hiriendo varios de ellos el cuerpo del animal. La flecha 11 penetró $8,2,1,1$ y $2 \mathrm{~cm}$ en los sucesivos disparos. La flecha 7 penetró en la oveja $25,4,8$ y $7 \mathrm{~cm}$ limpiamente, reduciendo su impacto a 6 y $2 \mathrm{~cm}$ dentro del cuerpo tras atravesar el peto de cuero. La media de disparos efectuados por flecha es de 5,4 para el conjunto de ellas y de 7,8 para las no recocidas (Fig. 4A, B).

El uso de las jabalinas estuvo condicionado por la poca experiencia de los participantes en el manejo de esta arma. En general, han podido ser utilizadas en un número de disparos más alto, si bien las empleadas con éxito fueron muchas menos que en el caso de las otras dos armas (media de aciertos total 1,1 frente a 1,8 de las flechas y 9 de las lanzas). 

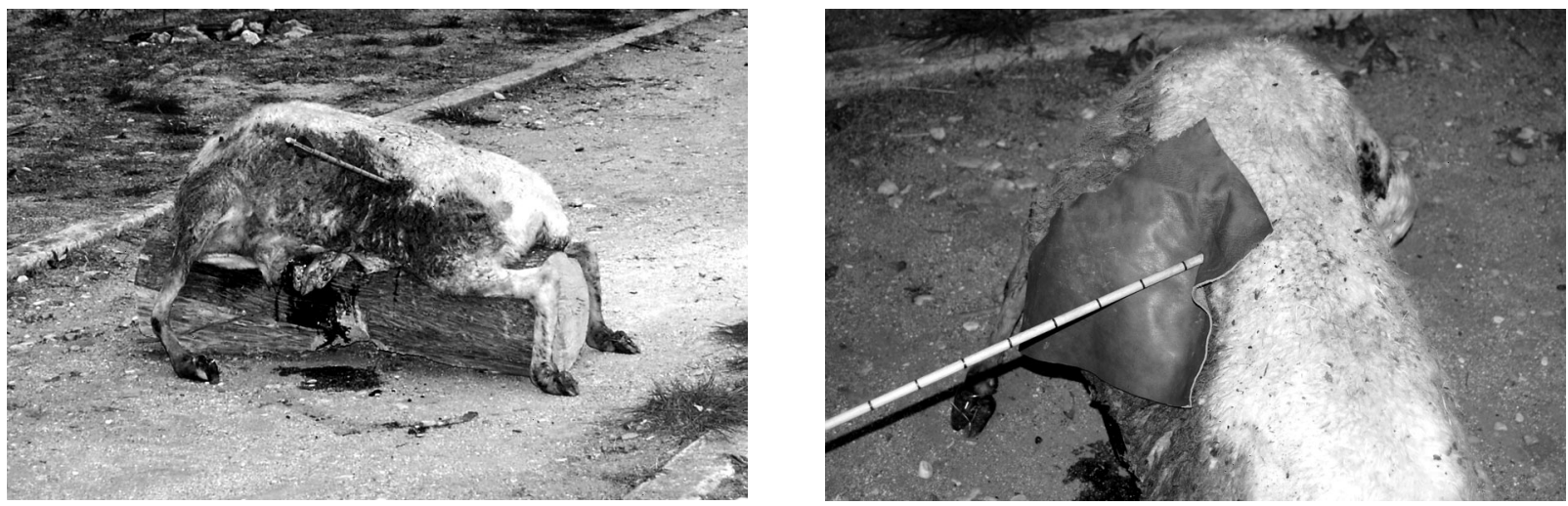

Fig. 4.A y B: Detalle de flecha 7 clavada en la oveja con y sin peto.

Casos excepcionales de resistencia suponen las puntas 10 de arco junto con la 17 y 18 de jabalina, únicamente forjadas. Todas ellas se clavaron en el tronco de madera que sostenía a la oveja, llegando a incrustarse la mitad de la hoja (Fig. 5A). En los tres ejemplares, las puntas fueron extraídas de la madera sin apenas daños y se continuó disparando con ellas. Por el contrario, cuando la punta 1 , forjada y recocida, clavada en la madera tras el primer lanzamiento, quedó doblada por la mitad y fuera del enmangue.

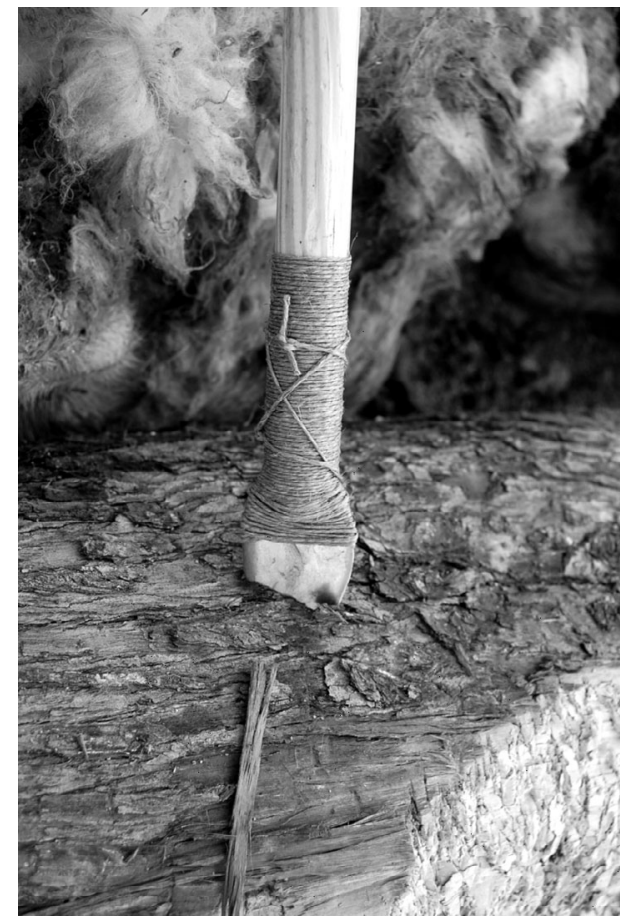

Fig. 5.A. Jabalina 17 clavada en madera.
Las lanzas, utilizadas a modo de pica, sufrieron distintos efectos. La oveja ya había pasado el rigor mortis al inicio de los experimentos pero empezaba a endurecerse, por lo que no resultó fácil clavarle las palmelas. Sin embargo, cuando las puntas penetraron en el cuerpo su efecto fue devastador ya que rompieron distintos huesos, sobre todo en la zona del costillar (Fig. 5B). Especialmente útil resultó la pieza 24 con la que se impactó en el animal hasta en 23 ocasiones, se falló en dos tiros debido a que se había sobrepuesto a la oveja el peto de cuero de $2 \mathrm{~mm}$.

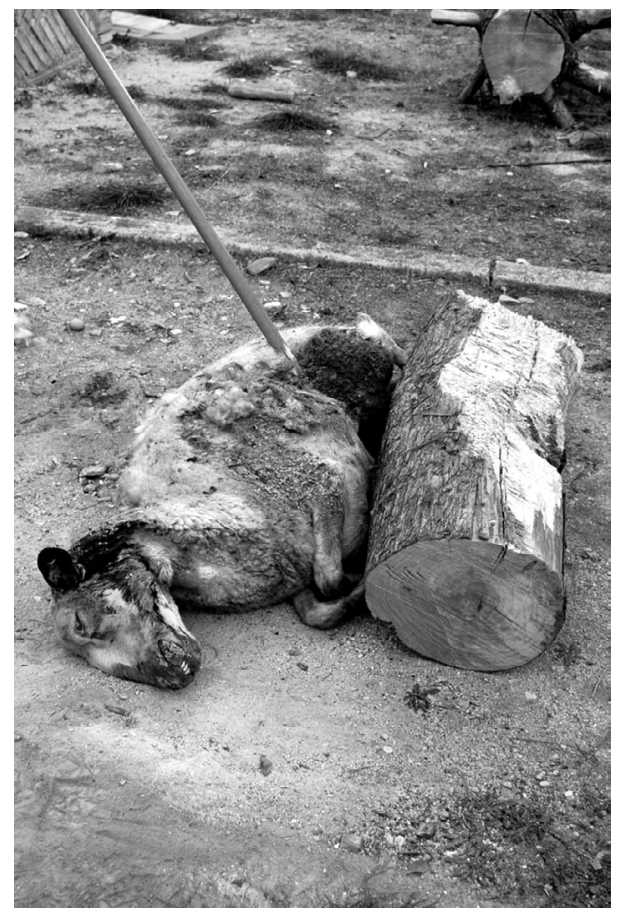

Fig. 5.B. Lanza 24 clavada en la oveja.

T. P., 67, N. ${ }^{\circ}$ 2, julio-diciembre 2010, pp. 405-418, ISSN: 0082-5638 doi: $10.3989 /$ tp.2010.10047 
Después de los experimentos recuperamos las puntas 4, 13, 16 y 17 que habían quedado dobladas por el uso. El objetivo era ponerlas nuevamente a punto aplanándolas por forja y limándolas de nuevo. Se invirtió unos 15 segundos en el enderezamiento y de 2 a 4 minutos en el afilado. Sobre algunas de las piezas quedaron unas marcas características, detectables a simple vista, que indican que la pieza había estado doblada.

\section{Pruebas de distancia}

Las pruebas de distancia las llevamos a cabo en el campo de rugby de la Universidad Autónoma de Madrid cuyo eje mayor tiene una longitud de $125 \mathrm{~m}$. Las armas fueron usadas por un único tirador para el arco y otro para la jabalina. Los resultados han sido dispares. Las distancias medias obtenidas con la jabalina son muy bajas y las diferencias no se corresponden con la modificación de variables. Parece que, en este caso, ha sido determinante la poca experiencia en el uso del arma.

Con las flechas se llevaron a cabo dos modalidades de tiro: con ángulo para tratar de obtener el máximo de distancia y tiro recto. El primer disparo con ángulo de la pieza 25 traspasó los $125 \mathrm{~m}$ del campo, de hecho, sobrevoló un montículo adyacente y quedó clavada profundamente en la tierra próxima a la acera de acceso a la UAM, la punta no pudo ser recuperada. Dado lo peligroso del experimento, tiramos las siguientes flechas -26 a 28 - con tiro recto. La longitud media alcanzada por la palmela pequeña supera bastante a los conseguidos con las piezas medianas, si bien no llega a duplicarlos. En las medianas se observan pocas diferencias entre las fabricadas en cobre y en bronce, siendo esta última la que menos longi- tud alcanzó. Estos resultados son coherentes con los ofrecidos por la balística. Sí observamos que, durante el vuelo, las flechas pequeñas se estabilizaron enseguida mientras que las medianas y la grande, tardaron más. Fuera del programa quisimos hacer un experimento más. Lanzadas ya las jabalinas, desmontamos la punta 32, grande, y la encajamos en un vástago de flecha profundizando en la caja. Efectuamos con ella 5 disparos de arco con ángulo y alcanzamos una distancia media de 79,59 m longitud, notable teniendo en cuenta que la punta pesa 40,26 gr. Este resultado no es comparable con los anteriores porque la modalidad de tiro, en ángulo o recto, ha sido distinta, pero nos muestra la eficacia de estas puntas incluso en aquellos ejemplares más pesados.

\section{Pruebas de balística}

Del segundo conjunto experimental, reservamos 4 piezas de distintas características para el estudio de balística. Éste se llevó a cabo en la Sala de tiro de la Policía Científica de Madrid. Se utilizó un medidor de velocidad (Drello \& BAL4042 V-computer) que da los resultados en metros por segundo a través de dos celdas fotoeléctricas distantes entre sí $1.128 \mathrm{~mm}$, situándose el tirador a $3 \mathrm{~m}$ de la primera. Los disparos fueron efectuados con nuestro arco de fresno. Cada pieza fue disparada 5 veces y la velocidad que se ofrece es la media de dichos disparos (Tab. 4), (Fig. 6).

Los resultados revelan una mayor ligereza de las piezas más pequeñas, siendo especialmente veloz la pieza de cobre que alcanza hasta 41,20 $\mathrm{m} / \mathrm{s}$. Más lentas han resultado las puntas de flecha medianas, en torno a $28 \mathrm{~m} / \mathrm{s}$, si bien en este caso

\begin{tabular}{|c|c|c|c|c|c|c|c|}
\hline $\mathbf{N .}^{\circ}$ & $\begin{array}{c}\text { Materia } \\
\text { Prima }\end{array}$ & Tratamiento & Tamaño & Peso (gr) & Arma & $\begin{array}{c}\text { Total } \\
\text { disparos }\end{array}$ & Distancia media (m) \\
\hline 25 & $\mathrm{Cu}$ & $\mathrm{FF}$ & Pequeña & 5,02 & Flecha & 1 & +125 \\
26 & $\mathrm{Br} 2 \%$ & $\mathrm{~F}+\mathrm{FF}$ & Pequeña & 5,43 & Flecha & 5 & 77,02 \\
27 & $\mathrm{Cu}$ & $\mathrm{FF}$ & Mediana & 21,16 & Flecha & 5 & 48,18 \\
28 & $\mathrm{Br} \%$ & $\mathrm{~F}+\mathrm{FF}$ & Mediana & 23,42 & Flecha & 5 & 44,5 \\
29 & $\mathrm{Br} \%$ & $\mathrm{~F}+\mathrm{FF}$ & Mediana & 23,91 & Jabalina & 5 & 19,17 \\
30 & $\mathrm{Cu}$ & $\mathrm{FF}$ & Grande & 33,34 & Jabalina & 5 & 19,27 \\
31 & $\mathrm{Br} 2 \%$ & $\mathrm{~F}+\mathrm{FF}$ & Grande & 39,49 & Jabalina & 5 & 15,5 \\
$32 \mathrm{a}$ & $\mathrm{Br} \%$ & $\mathrm{~F}+\mathrm{FF}$ & Grande & 40,26 & jabalina & 5 & 18,5 \\
$32 \mathrm{~b}$ & $\mathrm{Br} \%$ & $\mathrm{~F}+\mathrm{FF}$ & Grande & 40,26 & Flecha & 5 & 79,59 \\
\hline
\end{tabular}

Tab. 3. Pruebas de distancia (F: Fundido, FF: Forja en frío, Cu: Cobre, Br: Bronce). 


\begin{tabular}{|c|c|c|c|c|c|c|}
\hline N. ${ }^{o}$ & M. Prima & Tratamiento & Medidas (mm) & Peso (gr) & Peso flecha & $\begin{array}{c}\text { Resultado } \\
(\mathrm{m} / \mathrm{s})\end{array}$ \\
\hline 33 & $\mathrm{Cu}$ & $\mathrm{F}+\mathrm{FF}$ & Pequeña: $45 \times 13 \times 1.2$ & 3,77 & 46,62 & 41,20 \\
\hline 34 & $\operatorname{Br} 2 \%$ & $\mathrm{~F}+\mathrm{FF}$ & Pequeña: 46 × 14 × 2 & 5,33 & 58,38 & 34,52 \\
\hline 35 & $\mathrm{Cu}$ & $\mathrm{F}+\mathrm{FF}$ & Mediana: $98 \times 28 \times 2.2$ & 18,94 & 70,43 & 28,87 \\
\hline 36 & $\operatorname{Br} 2 \%$ & $\mathrm{~F}+\mathrm{FF}$ & Mediana: $105 \times 27 \times 2.9$ & 23,41 & 73,96 & 28,34 \\
\hline
\end{tabular}

Tab. 4. Pruebas de balística (F: Fundido, FF: Forja en frío, Cu: Cobre, Br: Bronce).

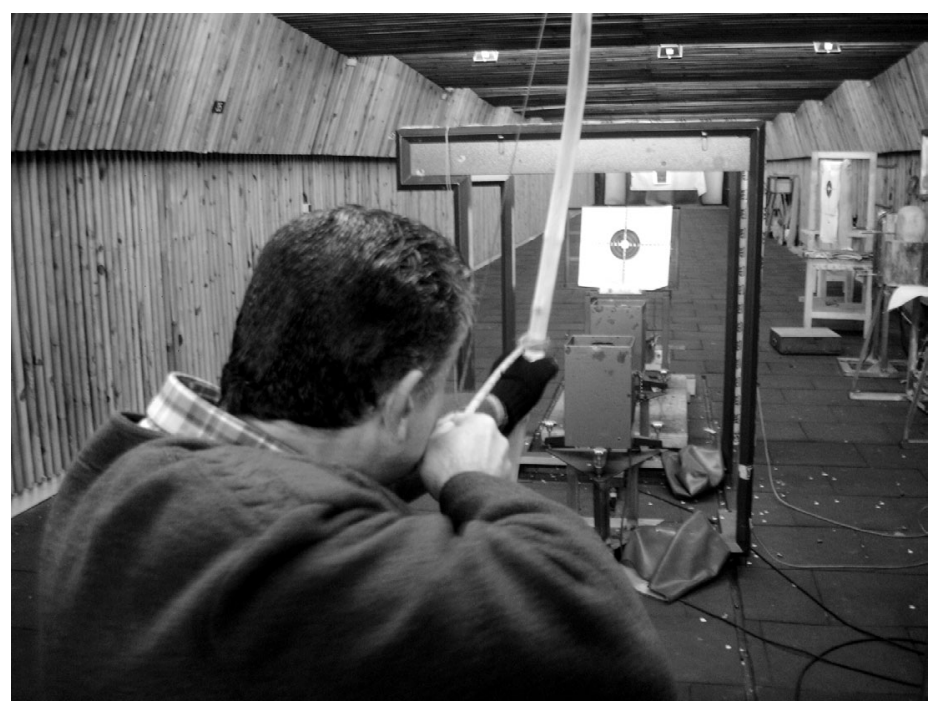

Fig. 6. Tiro con arco. Experimento de balística.

hay muy poca diferencia entre la de cobre y la de bronce.

Estos resultados pueden compararse a los recogidos por Quesada para las puntas usadas en el Reino Nuevo egipcio. Las flechas, tanto para la caza como para la guerra, pesaban entre 40 y 90 gr y se construían sobre caña, madera de acacia o tamarisco o una combinación de ambas. Además de puntas de madera de ébano o acacia se empleaban otras de cobre y bronce con nervio central y pedicelo largo. Estudios balísticos sobre piezas experimentales han arrojado resultados de 40 a $47 \mathrm{~m} / \mathrm{s}$ con arcos compuestos y $35 \mathrm{~m} / \mathrm{s}$ con arco simple doble-convexo (deflejo). En las puntas grandes de cobre la velocidad bajaba a $32 \mathrm{~m} / \mathrm{s}$. La potencia de los arcos era, aproximadamente, de 50 libras para el arco simple y hasta 64 libras en el compuesto (Quesada Sanz 2008: 178).

Teniendo en cuenta que nuestro arco es simple y con una potencia inferior a los egipcios, 35 libras, los resultados arrojados por nuestras flechas con puntas de palmela metálica son óptimos en ambos tamaños.

\section{DISCUSIÓN}

La eficacia del uso de las puntas de palmela es alta en las tres modalidades de arma. En especial, no vemos motivos para descartar el uso de las palmelas como extremos de flecha, en ninguno de sus tamaños y pesos. Por el contrario, sí cabe desechar que los ejemplares más pequeños fuesen empleados como puntas de jabalina o lanza, ya que los vástagos tendrían que ser extremadamente delgados para encajar los pedúnculos de forma eficaz, hecho que, por sí mismo, anularía su efectividad.

Uno de los aspectos más conflictivos de las puntas de palmela, y origen de su desacuerdo funcional, es la variación de tamaños y pesos. Esta circunstancia puede estar motivada por diferentes 
factores. Desde el punto de vista técnico la conformación mediante batido a partir de láminas no propicia obtener piezas homogéneas que se procurarían, únicamente, con el empleo de moldes. En el registro arqueológico los moldes para puntas de palmela se han evidenciado en el ejemplar valenciano del Gargao (Simón 1998: 164) y en los análisis metalográficos de la punta de Lituero (Rovira y Delibes 2005: 502).

Además, las sucesivas puestas en forma de los ejemplares, deteriorados por el uso continuado, diversificarían el conjunto morfométrico. Finalmente, la variedad puede proceder de las propias necesidades del uso. No todas las puntas son igualmente útiles en circunstancias diferentes. Aquellas de escaso peso con pluma ligera tienen más capacidad de penetración y son apropiadas para trayectorias largas. Por el contrario, si se requiere mayor poder de parada en distancias más cortas, la punta deberá ser pesada y con un emplumado largo (A. Romo y D. Bermejo comunicación personal). Así, para herir a un animal o enemigo alejado, la palmela pequeña es más eficaz, pero para rematar a corta distancia es preferible una punta más grande, que infringe mayor destrozo. Este hecho pudo constatarse muy tempranamente seleccionándose el tipo de flecha en función de su uso.

Una explicación común para desechar a las palmelas como puntas de flecha es su excesivo peso. Respecto a esto, ha quedado patente que las puntas pequeñas y medianas, que incluyen pesos de 4 a 22 gr, son perfectamente aptas como flechas, así lo indican los experimentos sobre la oveja y la balística. Por razones de seguridad, no pudimos emplearlas en las pruebas de distancia disparando en ángulo, donde hubieran logrado su mayor recorrido. La única prueba como flecha con una punta grande obtuvo distancias notables para una pieza de 40 gr. Todos los experimentos se han llevado a cabo con un arco simple de 35 libras. Un arco más potente y/o reforzado permitiría alcanzar aún mejores resultados. Por otra parte, las piezas mayores de $25 \mathrm{gr}$ son las menos frecuentes en el registro arqueológico, centrándose el grueso de palmelas entre 4 y 24 gr (según el catálogo de Rovira et al. 1997), lo que equivale, prácticamente, a nuestras puntas pequeñas y medianas. El empleo como cabezas de flecha de ejemplares pesados ha sido puesto de manifiesto también por Blas Cortina y Rovira Llorens (2006: 291-292) a par- tir del ejemplar de Dureyu, con 18,3 g de peso y marcas de impacto en su punta. Argumentan estos autores que además del peso y tamaño son determinantes las dimensiones y diseño del arco, así como la forma de montaje en el astil y la proporción de este último. Igualmente plantean que no sería extraño que los arqueros de la antigüedad llevasen flechas de diversos tamaños que elegirían en función de su objetivo.

Se ha constatado en diversos yacimientos la aparición de palmelas junto a puntas líticas, sugiriéndose la idea de la superioridad de las segundas y el valor, más simbólico que funcional, de las metálicas (Delibes y Santiago 1997: 103, 107). Abundan en esta opinión Fernández Manzano y Montero (1997: 111-113), si bien recuerdan los hallazgos del cráneo de Grajal de los Campos, con dos puntas de palmela y de la Cova de l'Heura de Arboli, en Tarragona, citado por Campillo (1977: 290-295), donde se recogió un cráneo con una punta pedunculada triangular incrustada. Un tercer ejemplo del uso de estas piezas apareció sobre otro individuo del Dolmen de Collet Sú en Lérida, en cuyo parietal izquierdo se alojaba el ápice de una punta metálica, en este caso con signos de cicatrización (Campillo 1977: 290-295, 1983: 57).

La supuesta poca eficacia de los primeros útiles/armas metálicos se apoya en la escasa dureza del cobre, incluso del cobre arsenicado, y en su facilidad para deformarse. Cabe insistir en que el cobre y, aún más, el bronce con contenidos muy bajos de estaño, convenientemente forjado, si bien no es del todo equiparable a un buen bronce, incrementa notablemente la dureza del metal y se transforma en una materia apta como punta de arma arrojadiza o de lanza, convirtiéndose así en una alternativa a la piedra para la fabricación de puntas. A este hecho hay que añadir la facilidad de reavivado, ya que el metal se deforma antes que se quiebra y la nueva puesta en uso es rápida y eficaz. En este aspecto, el metal aventaja insuperablemente a la piedra. Las puntas de flecha talladas sobre materiales silíceos deben desecharse al fragmentarse, salvo que la rotura sea mínima, e incluso, durante el retoque de fabricación y/o reavivado hay riesgo de ruptura por su gran fragilidad. Su superioridad reside en una materia prima más accesible y de pronta fabricación. No todas son talladas sobre sílex de calidad mediante los bellos retoques en peladura, ya que en el registro arqueológico abundan las puntas con retoques 
más irregulares, producto de una manufactura expeditiva, incluso sobre materias pobres pero eficaces, como pizarras.

No creemos que la cuestión se centre en la superioridad de una materia sobre otra. Ambas han mostrado su validez y oponen la rapidez y facilidad de fabricación de la lítica al mejor mantenimiento de las metálicas. Cazadores y/o guerreros pudieron usar ambas en función de su accesibilidad, su urgencia y sus objetivos. La asociación de puntas de piedra y metal en los enterramientos campaniformes no invalida la eficacia de las de metal sino que sugiere que ambas eran tenidas en alta consideración como armamento.

Durante los inicios de la metalurgia ha habido una larga fase en que hachas, punzones o sierras en piedra, hueso y metal conviven. Los primeros objetos metálicos copian modelos bien establecidos en las otras materias. Las palmelas no son una excepción. Reproducen las morfologías foliáceas de algunos tipos líticos y añaden un pedúnculo más largo, inviable en la piedra por su fragilidad. Esta modificación supone una cierta capacidad de experimentación que consigue diseños simples pero aerodinámicos de armamento con una tecnología metalúrgica poco evolucionada a partir de cobre solo o arsenicado. El proceso de ensayo continuará en los ejemplares más modernos con la adición de un engrosamiento o nervio central ancho que aporta robustez a la hoja y evita su doblamiento. En este mismo sentido, Delibes et al. (1999: 71) apuntan que durante la fase campaniforme se produce una renovación del armamento con la aparición de las puntas de palmela y los puñales de lengüeta, pero no de los instrumentos domésticos. La innovación que aporta la palmela puede referirse a la fabricación de puntas de flecha metálicas pero no hay que descartar que incluyese nuevas tentativas de armas como la jabalina o la lanza de punta metálica, si bien se ha sugerido el empleo de puñales para esta función (Simón 1999: 204).

Sin embargo, la punta de flecha, al menos de piedra, es el único armamento bien constatado, y tipificado, en el registro arqueológico desde el Neolítico al Bronce antiguo. Se documentan en las escenas de caza y guerra del arte levantino y en los ejemplos de puntas líticas clavadas en esqueletos neolíticos y calcolíticos de San Juan ante Portam Latinam, Bóvila Madurell, Longar y Camí de Can Grau (Isidro y Malgosa 2003). Cabe añadir las saeteras en las murallas del poblado de los Millares (Molina y Cámara 2005), entre otros de esta última cronología. Es posible que, junto con puntas de flecha, se utilizasen armas como mazas, báculos o boomerangs, puñales, alabardas y hachas, líticas y metálicas (Mederos 2009). El registro es parco en evidencias y las que más abundan siguen siendo las puntas de flecha (Delibes y Santiago 1997: 103), lo que aporta un argumento a favor del empleo de las palmelas como extremos de flecha. Posteriormente, a partir del Bronce medio, se consolidan tipos más reducidos y livianos de puntas de flecha metálicas, con formas como las de pedúnculo y aletas o únicamente pedunculadas. El desarrollo de estas armas, en paralelo a la aparición de las espadas, supone una mejora de la tecnología metalúrgica (Rovira et al. 1992: 278) y también una posible renovación de las tácticas de combate que impulsaría el uso de elementos más ligeros.

La experimentación ha demostrado la eficacia de las puntas de palmela montadas sobre armas como flecha, jabalina o lanza. Esto nos permite sugerir algunas matizaciones acerca de su uso. Somos conscientes de que, dada la amplitud del trabajo, no se han agotado todas las variables posibles quedando abiertas otras aplicaciones de tipo experimental. El paso siguiente será el estudio de las huellas dejadas por cada uso, que se deberá completar con el análisis de una colección arqueológica. Sólo la contrastación de huellas experimentales y arqueológicas, junto al análisis del contexto, nos permitirá aproximarnos, de modo más preciso, a la función de este tipo de piezas.

\section{AGRADECIMIENTOS}

Al personal del Departamento de Armas de la Dirección General de la Policía del Ministerio del Interior (Madrid), donde se llevó a cabo el estudio de balística. A Miguel Ángel Varona, Director del Servicio, Daniel Bermejo, José Daniel Gómez y, de forma muy especial, a Ángel Romo, que actuó como arquero experimentado. También han sido de gran ayuda Juan Francisco Blanco, por sus consejos y la foto inédita de Coca, Belén Márquez, que colaboró en la experimentación de balística y Fernando Quesada por su asesoramiento sobre armamento antiguo. A todos ellos muchas gracias. 


\section{BIBLIOGRAFÍA}

Blas Cortina, M.A. y Rovira Llores, S. 2006: "Huellas de actividad prehistórica en un medio montañoso extremo: en torno a una palmela en la Garganta del Cares, Picos de Europa (Asturias)". Munibe 57: 287-299.

Briard, J. y Mohen, J.P. 1983: "Typologie des objets de l'Age du Bronze en France". Fascicule II. Poignards, hallebardes, pointes de lance, pointes de flèche, armement défensif. Société Préhistorique Française. Paris.

Campillo, D. 1977: Paleopatología del cráneo en Cataluña, Valencia y Baleares. Montblanc-Martín. Barcelona.

Campillo, D. 1983: La enfermedad en la Prehistoria. Introducción a la Paleopatología. Salvat. Madrid.

Clark, J.G.D. 1963: "Neolithics bows Somerset, England, and the prehistory of archery in NorthWest Europe". Proceedings of the Prehistoric Society 29: 50-98.

Delibes de Castro, G. 1977: El Vaso campaniforme en la meseta norte española. Studia Archaeologica 46. Universidad de Valladolid. Valladolid.

Delibes de Castro, G.; Fernández Manzano, J. y Herrán Martínez, J.L. 1999: "Submeseta Norte". En G. Delibes de Castro e I. Montero Ruiz (coords.): Las primeras etapas metalúrgicas en la Península Ibérica II. Estudios regionales. Instituto Universitario Ortega y Gasset, Ministerio de Cultura. Madrid: 63-94.

Delibes de Castro, G. y Fernández-Miranda, M. 1981: "La tumba de Celada de Roblecedo (Palencia) y los inicios del Bronce antiguo en el valle medio y alto del Pisuerga". Trabajos de Prehistoria 38: 153-188.

Delibes de Castro, G. y Santiago Pardo, J. 1997: “Las fortificaciones de la Edad del Cobre en la Península Ibérica”. En J.A. García Castro, V. Antona del Val y L. Azcue Brea (coords.): La guerra en la Antigüedad. Una aproximación al origen de los ejércitos en Hispania. Ministerio de Defensa. Madrid: 85-108.

Fernández Manzano, J. y Montero Ruiz, I. 1997: “Las armas durante el Calcolítico y la edad del Bronce”. En J.A. García Castro, V. Antona del Val y L. Azcue Brea (coords.): La guerra en la Antigüedad. Una aproximación al origen de los ejércitos en Hispania. Ministerio de Defensa. Madrid: 109-122.

Garrido-Pena, R. 2000: El Campaniforme en La Meseta Central de la Península Ibérica (c. 2500-2000 a.C.). British Archaeological Reports International Series 892. Archaeopress. Oxford.

Gutiérrez Sáez, C. y Soriano Llopis, I. 2008: “La funcionalidad sobre material metálico. Bases y aplica- ciones de estudio". Actas del VII Congreso Ibérico de Arqueometría: 432-447. Madrid.

Hernando Grande, A. 1992: Materiales metálicos de la edad del Bronce en la Meseta: Armas. Cuadernos de la UNED. Madrid.

Herrán Martínez, J.L. 2008: Arqueometalurgia de la Edad del Bronce en Castilla y León. Universidad de Valladolid, Junta de Castilla y León. Valladolid.

Hunt, M. y Hurtado, V. 1999: "Suroeste". En G. Delibes de Castro e I. Montero Ruiz (coords.): Las primeras etapas metalúrgicas en la Península Ibérica II. Estudios regionales. Instituto Universitario Ortega y Gasset, Ministerio de Cultura. Madrid: 275-334.

Isidro, A. y Malgosa, A. 2003: Paleopatología. La enfermedad no escrita. Masson. Barcelona.

Káiser, J.M. a 2003: "Puntas de flecha de la Edad del Bronce en la Península Ibérica. Producción, circulación y cronología". Complutum 14: 73-106.

Mederos, A. 2009: "El Calcolítico". En H. O’Donnell (dir.): Historia Militar de España. I.M. Almagro Gorbea (ed.): Prehistoria y Antigüedad. Ediciones del Laberinto-Ministerio de Defensa. Madrid: 33-48.

Méndez Madariaga, A. 1997: "El arco como arma de guerra en la Antigüedad”. En J.A. García Castro, V. Antona del Val y L. Azcue Brea (coords.): La guerra en la Antigüedad. Una aproximación al origen de los ejércitos en Hispania. Ministerio de Defensa. Madrid: 63-70.

Molina, F. y Cámara, J.A. 2005: Los Millares. Dirección General de Bienes Culturales. Sevilla.

Muñoz Ibáñez, F.J. y Ripoll López, S. 2006: "Las primeras evidencias de arcos en Europa: Parámetros de diseño y construcción". En J.M. Maíllo y E. Baquedano (coords.). Miscelánea en homenaje a Victoria Cabrera. Zona Arqueológica 7. Madrid: 462-473.

Quesada Sanz, F. 2008: "El arco en el antiguo Egipto". La aventura de la Historia 121: 176-178.

Rodríguez de la Esperanza, M. ${ }^{a} J .1999:$ “Aragón”. En G. Delibes de Castro e I. Montero Ruiz. (coords.): Las primeras etapas metalúrgicas en la Península Ibérica. III Estudios regionales. Fundación Ortega y Gasset, Ministerio de Cultura. Madrid: 95-114.

Rovira Llorens, S. y Delibes de Castro, G. 2005: "Tecnología metalúrgica campaniforme en la Península Ibérica. Coladas, moldeado y tratamientos postfundición”. En M.A. Rojo Guerra, R. Garrido Pena e I. García Martínez de Lagrán (coords.): El campaniforme en la Península Ibérica y su contexto europeo. Universidad de Valladolid, Junta de Castilla y León. Valladolid: 495-512.

Rovira Llorens, S. y Gómez Ramos, P. 2003: Las Primeras etapas metalúrgicas en la Península Ibérica. III Estudios metalográficos. Instituto Universitario Ortega y Gasset, Ministerio de Cultura. Madrid. 
Rovira, S.; Montero, I. y Consuegra, S. 1992: “Archaeometallurgical study of Palmela arrow heads and other related types". En E. Antonacci (ed.): Archeometallurgia richerche e prospettive. Atti del Colloquio Internazionale di Archeometallurgia (Bologna-Dozza Imoleste, $18-21$ ottobre 1988). Bolonia: 269-289.

Rovira Port, J. 1998: "Una punta de jabalina asimilable al tipo palmela procedente de Benquerencia (Badajoz)". Cuadernos de Prehistoria y Arqueología de Castellón 19: 127-130.

Simón García, J.L. 1998: La metalurgia prehistórica valenciana. Diputación provincial de Valencia. Valencia.

Simón García, J.L. 1999: "Comunidad Valenciana". En G. Delibes de Castro e I. Montero Ruiz. (coords.): Las primeras etapas metalúrgicas en la Península Ibérica. III Estudios regionales. Fundación Ortega y Gasset, Ministerio de Cultura. Madrid: $172-216$.
Spindler, K. 1995: El hombre de los hielos. Círculo de Lectores. Madrid.

Los autores de las fotografías somos los firmantes del trabajo indistintamente.

Proyecto de referencia: Proyecto 06/0133/2002: Traceología sobre material metálico. Metodología de estudio y aplicación a instrumentos metálicos del Museo Arqueológico Nacional. Convocatoria Ayudas. Proyectos de Investigación Humanidades, Ciencias Sociales y Económicas Año 2002. Comunidad de Madrid. Duración: 12 meses. Investigadora principal C. Gutiérrez Sáez. 